\title{
Evaluation of GPS L5 and Galileo E1 and E5a Performance for Future Multi Frequency and Multi Constellation GBAS
}

\author{
M.-S. Circiu, M. Felux, S. Thölert, F. Antreich, M. Vergara, M. Sgammini, C. Enneking German Aerospace Center - DLR
}

\author{
S. Pullen Stanford University
}

\begin{abstract}
In this paper, we show a performance analysis of different signals from the new Galileo satellites in the E1 and E5a frequency bands as well as GPS L5 signals in DLR's experimental Ground Based Augmentation System (GBAS). We show results of noise and multipath evaluations of the available Galileo satellites and compare their performance to the currently used GPS L1 and the new GPS L5 signals which were presented in a recent paper. The results show that the raw noise and multipath level of Galileo signals is smaller than of GPS. Even after smoothing, Galileo signals perform somewhat better than GPS and are less sensitive to the smoothing time constant.
\end{abstract}

Another issue to be considered in a future multi frequency system is inter-frequency bias. These biases differ between satellites and depend on satellite and receiver hardware, but they can be determined a priori. With known receiver and antenna configurations, it is possible to correct for these biases and avoid errors introduced by different hardware in the airborne receiver and GBAS ground system. A residual uncertainty associated with the bias correction has to be taken into account. This can be modelled as part of $\sigma_{\text {pr_gnd }}$.

\section{INTRODUCTION}

In the last couple of years, several GBAS ground stations (Sydney, Malaga, Frankfurt and Zurich) have become operational and are used on a regular basis for approach guidance. These stations are so-called GBAS Approach Service Type C (GAST C) stations and support approaches only under CAT-I weather conditions; i.e., with a certain minimum visibility. Standards for stations supporting CAT-II/III operations (called GAST D), are expected to be agreed upon by ICAO later this year and stations could be commercially available as soon as 2018 . However, for both GAST C and D, the availability of the GBAS approach service can be significantly reduced under active ionospheric conditions. One potential solution is the use of two frequencies and multiple constellations in order to be able to correct for ionospheric impacts, detect and remove any compromised satellites, and improve the overall satellite geometry (and thus the availability) of the system.

A new multi frequency and multi constellation (MFMC) GBAS will have different potential error sources and failure modes which have to be considered and bounded. Thus, all performance and integrity assumptions of the existing single frequency GBAS must be carefully reviewed before they can be applied to an MFMC system. A central element for ensuring the integrity of the estimated position solution is the calculation of protection levels. This is done by modeling all disturbances to the navigation signals in a conservative way and then estimating a bound on the resulting positioning errors that is valid at an allocated integrity risk probability.

One of the parameters which is different for the new signals and has to be re-characterized is the residual uncertainty attributed to the corrections from the ground system $\left(\sigma_{p r_{-} g n d}\right)$. The contribution of residual noise and multipath is estimated by evaluating the B-values in GBAS, which give an estimate of the error contribution from a single reference receiver to a broadcast correction. Independent data samples over at least one day (for GPS) are collected and sorted by elevation angle. Then the mean and standard deviations for each elevation bin are determined.

In a recent paper [1], we presented these evaluations for GPS L5. Here, we extend the evaluation to the E1 and E5a signals broadcast by the operational Galileo satellites now in orbit. In the same manner as for GPS L5, we determine the $\sigma_{p r_{-} g n d}$ values for these Galileo signals. As for GPS L5, results show a lower level of noise and multipath in unsmoothed pseudorange measurements compared to GPS L1.

Another aspect which needs to be considered is the impact of inter-frequency biases. These biases are dependent on the analog and digital distortions of signals on different frequencies on each satellite as well as user receiver parameters (correlator spacing and receiver bandwidth), signal processing methods, and receiver front-end filtering and antenna characteristics. The respective inter-frequency biases for each satellite and 


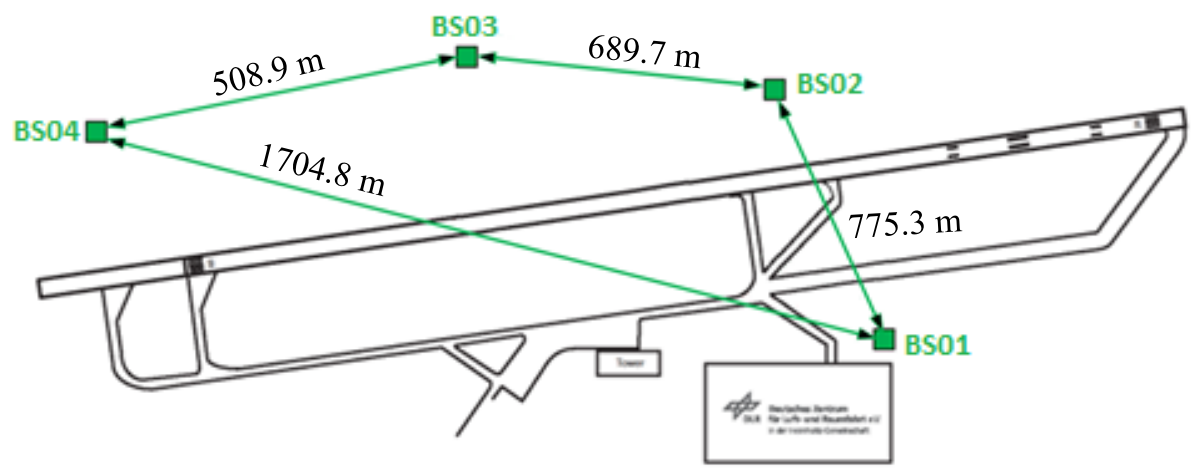

Figure 1. DLR Ground Facility close to Braunschweig Airport

frequency-band combination can be derived using high gain antenna measurements, joint estimation of analog and digital distortions, and derivation of code and phase tracking error bias and variance using appropriate receiver models. Such an analysis can be extended to all Galileo and GPS satellites. With this knowledge, the interfrequency biases can be largely removed and differential errors due to different hardware in the GBAS ground station and the airborne system reduced significantly.

In this paper, we discuss both of these issues (noise/multipath and inter-frequency bias) as contributions to $\sigma_{p r}$ gnd . After a brief description of our GBAS test bed and a review of the different smoothing techniques, we present results of noise and multipath analysis of the new signals. Next, we assess their performance in a GBAS context; i.e., after applying the different smoothing methods with varying smoothing time constants. Then we explain the problem of interfrequency biases and show a proposal for how to account for them in a multi frequency GBAS and how to model the residual uncertainty for integrity purposes.

\section{DLR GBAS GROUND FACILITY}

DLR has set up a GBAS prototype at the research airport in Braunschweig (ICAO identifier EDVE) near the DLR research facility there. This ground station has recently been updated and now consists of four Javad Delta receivers connected to Leica AR 25 choke ring antennas which are mounted at heights between 2.5 meters and 7.5 meters above equipment shelters. All four receivers are capable of tracking GPS L5 (in addition to GPS L1 and L2 semi-codeless) and Galileo E1 and E5a signals. Figure 1 shows an overview of the current ground station layout, and the coordinates of the antennas are presented in Table 1.

Table 1. Ground receiver antenna coordinates

\begin{tabular}{|c|c|c|c|}
\hline Receiver & Latitude $\left[{ }^{\circ}\right]$ & Longitude $\left[{ }^{\circ}\right]$ & Height [m] \\
\hline BS01 & $52^{\circ} 19^{\prime} 2^{\prime \prime} \mathrm{N}$ & $10^{\circ} 34^{\prime} 2^{\prime \prime} \mathrm{E}$ & 134.21 \\
\hline BS02 & $52^{\circ} 19^{\prime} 6^{\prime \prime} \mathrm{N}$ & $10^{\circ} 33^{\prime} 5^{\prime \prime} \mathrm{E}$ & 137.53 \\
\hline BS03 & $52^{\circ} 19^{\prime} 20^{\prime \prime} \mathrm{N}$ & 10³3'16’'E & 133.25 \\
\hline BS04 & $52^{\circ} 19^{\prime} 17^{\prime \prime} \mathrm{N}$ & $10^{\circ} 32^{\prime} 36^{\prime \prime} \mathrm{E}$ & 131.51 \\
\hline
\end{tabular}

\section{SMOOTHING TECHNIQUES IN GBAS}

The GBAS system corrects for the combined effects of multiple sources of measurement errors that are highly correlated between reference receivers and users such as satellite clock, ephemeris error, ionospheric delay error, and tropospheric delay error through the differential corrections broadcasted by the GBAS ground subsystem. However, uncorrelated errors such as multipath and receiver noise can make a significant contribution to the remaining differential error. Multipath errors are introduced by the satellite signal reaching the antenna via both the direct path from the satellites and from other paths due to reflection or diffraction. These errors affect both the ground and the airborne receivers but are different at each and do not cancel out when differential corrections are applied. In order to reduce these errors, GBAS performs carrier smoothing. Smoothing makes use of the less noisy but ambiguous carrier-phase measurements to suppress the noise and multipath from the noisy but unambiguous code measurements.

The current GBAS architecture is based on single frequency GPS L1 C/A code measurements only. With the new available satellites (GPS Block IIF and Galileo) broadcasting in an additional aeronautical band (L5 / E5), this second frequency could be used in GBAS to overcome many current limitations of the single frequency system. Single frequency carrier smoothing reduces noise and multipath, but ionospheric disturbances can cause significant differential errors when the ground station and the airborne user are affected by different conditions. Dual frequency techniques have been investigated in previous work [4]. Two dual frequency smoothing algorithms, Divergence Free (Dfree) and Ionosphere Free (Ifree), have been proposed to mitigate the effect of ionosphere gradients.

All GNSS smoothing techniques make use of a low pass filter, but the inputs are different. In general, the input to the filter is the difference of the code input $\left(\rho_{i n}\right)$ and carrier-phase input $\left(\phi_{\text {in }}\right)$, which is called "code minus carrier” or CMC. The final smoothed pseudoranges are obtained by adding the carrier-phase measurements back into the smoothed CMC. Figure 2 shows a representation of a generic smoothing filter, where $\rho_{\text {in }}$ is the code 
measurement input and $\phi_{\text {in }}$ is the carrier-phase measurement input.

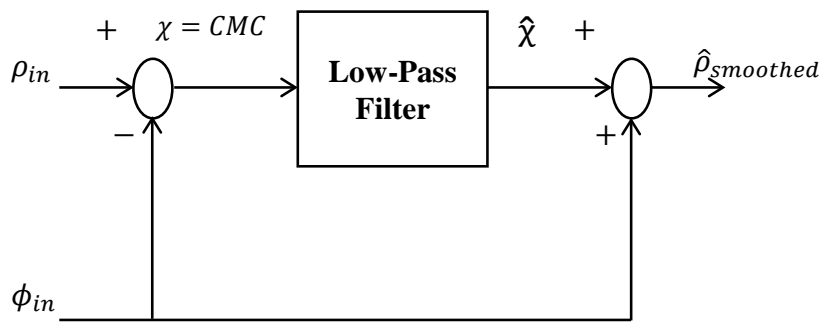

Figure 2. Generic Low pass smoothing filter

\section{Single frequency smoothing}

In single frequency smoothing, code and carrier-phase measurements from the same signal frequency are input to the filter. Since the ionosphere affects code and carrier measurements by the same amount but with opposite sign, the filter input will contain double the ionospheric delay, as expressed in equation (1), where $\chi$ is the filter input.

$$
\chi=\rho_{i}-\phi_{i}=2 I_{i}-N_{i}+\epsilon_{i}
$$

In this equation, $I$ represents the ionospheric delay, $N$ the carrier-phase integer ambiguity, and $\epsilon$ the code noise (carrier phase error is neglected, as it is much smaller than the code noise error). The coefficient $i$ refers to signal frequency $i$.

After recombining the ranging information by adding the carrier input to the output of the filter, the carriersmoothed code measurement is expressed by the following (theoretical) equation:

$$
\hat{\rho}_{\text {smoothed }}=r+\epsilon_{i, \text { smoothed }}+\left(2 I_{i, \text { smoothed }}-I_{i}\right)
$$

where $r$ represents the geometric range from user to the satellite, $\epsilon_{i, \text { smoothed }}$ the smoothed noise on code, $I_{i}$ the (unknown) ionospheric error on the raw code measurement, and $I_{i, \text { smoothed }}$ the ionospheric error on code after smoothing. If the ionospheric error is constant over time, the low-pass filter would not have any impact on it, and $I_{i \text {,smoothed }}=I_{i}$. Since the ionosphere generally varies with time, the single frequency filter introduces an additional delay due to the difference between its (averaged) impact on smoothed code and its (unaffected) impact on carrier. This effect is called "code-carrier divergence."

\section{Divergence-free Smoothing (Dfree)}

Divergence-free smoothing ([7]) eliminates ionospheric delay from the filter input and thus removes the codecarrier divergence effect. This is achieved by using a linear combination of dual-frequency carrier phase measurements as the carrier input into the smoothing filter. The expression for the carrier phase input is described in equation (3), where the resulting phase
$\phi_{D \text { free }}$ replaces $\phi_{\text {in }}$ as the carrier filter input in Figure 1. The ionospheric delay created by combining the carrier phase measurements has the same sign and magnitude as the one in the code measurements. The code input is the raw code from frequency $i$, as in the single frequency case (see equation (4)):

$$
\begin{aligned}
& \phi_{D f r e e}= \phi_{i}-\frac{2}{\alpha}\left(\phi_{i}-\phi_{j}\right)= \\
&=r+I_{i}+N_{i}-\frac{2}{\alpha}\left(N_{i}-N_{j}\right) \\
& \text { with } \alpha=1-f_{i}^{2} / f_{j}^{2}
\end{aligned}
$$

$\rho_{\text {Dfree }}=\rho_{i}$

Equation (5) describes the smoothed Dfree ranges.

$\rho_{\text {Dfree_smoothed }}=r+I_{i}+\epsilon_{i, \text { smoothed }}$

The Dfree output removes the temporal ionospheric gradient that affects the single frequency filter but is still affected by the absolute difference in delay created by spatial gradients. The main advantage of Dfree is that the output noise is similar to that of single frequency smoothing, since only one single frequency code measurement is used as the code input (recall that carrier phase noise on both frequencies is small and can be neglected). Another advantage is that the final Dfree smoothed pseudoranges do not contain inter-frequency biases. These biases are constant over time and are removed from the final smoothed pseudoranges when the phase combination input is added to the smoothed CMC.

\section{$\underline{\text { Ionosphere-free Smoothing (Ifree) }}$}

Ionosphere-free smoothing ([7]) completely removes the (first-order) effects of ionospheric delay by using ionosphere-free combinations of code and phase measurements from two frequencies as inputs to the smoothing filter. The inputs can then be written as

$$
\begin{aligned}
& \phi_{\text {Ifree }}=\phi_{i}-\frac{1}{\alpha}\left(\phi_{i}-\phi_{j}\right) \\
& \rho_{\text {Ifree }}=\rho_{i}-\frac{1}{\alpha}\left(\rho_{i}-\rho_{j}\right)
\end{aligned}
$$

The smoothed Ifree pseudoranges then become

$$
\begin{aligned}
\rho_{\text {Ifree_smoothed }}= & r+\epsilon_{i, \text { smoothed }} \\
& -\frac{1}{\alpha}\left(\epsilon_{i, \text { smoothed }}-\epsilon_{j, \text { smoothed }}\right) \\
& -\frac{1}{\alpha} I F B
\end{aligned}
$$

where $r$ is the range information, $\epsilon_{i, \text { smoothed }}$ and $\epsilon_{j, \text { smoothed }}$ are the smoothed errors on frequencies $i$ and $j$, respectively, and $I F B$ represents the inter-frequency bias caused by hardware differences between the two frequencies and appears when combining code measurements across frequencies. Unlike the Dfree solution, the IFB is not removed from the final smoothed 
pseudoranges and will affect the Ifree position solution, as this bias differs among satellites. Ifree outputs no longer contain ionospheric errors (to a first-order approximation), but they contain the combination of errors from two code measurements. This increases the standard deviation of the expected differential pseudorange error and thus also of the position solution.

\section{NOISE AND MULTIPATH PERFORMANCE OF NEW GNSS SIGNALS}

GBAS users compute nominal protection levels $\left(\mathrm{H}_{0}\right)$ under a fault free assumption. These protection levels are conservative overbounds of the maximum position error after application of the differential corrections broadcast by the ground system, assuming that no faults or anomalies affect the position solution. In order to compute these error bounds, the total standard deviation of each differentially corrected pseudorange measurements has to be modeled. The standard deviation of the residual uncertainty ( $\sigma_{n}$, for the $n$th satellite) consists of the root-sum-square of uncertainties introduced by atmospheric effects (ionosphere, troposphere) as well as of the contribution of the ground multipath and noise. In other words, these error components are combined to estimate $\sigma_{n}^{2}$ as described in the following equation:

$$
\sigma_{n}^{2}=\sigma_{p r_{-} g n d}^{2}+\sigma_{p r_{-} a i r}^{2}+\sigma_{\text {iono }}^{2}+\sigma_{\text {tropo }}^{2}
$$

The ground broadcasts a value for $\sigma_{p r_{-} g n d}$ (described later in the section) associated with the pseudorange correction for each satellite. These broadcast values are based on combinations of theoretical models and actual measurements collected from the ground receivers that represent actual system characteristics. Unlike the ground, $\sigma_{p r_{-} \text {air }}$ is computed based entirely on a standardized error model. This is mainly to avoid the evaluation of multipath for each receiver and each aircraft during equipment approval.

In addition to the characteristics of nearby signal reflectors, multipath errors are mainly dependent on signal modulation and other signal characteristics (e.g., power, chip rate). In a previous paper [6], we showed that the newly available L5 signals broadcast by the GPS Block IIF satellites show better performance in terms of lower noise and multipath. This mainly results from an increased transmitted power and a 10 times higher chip rate on L5 compared to the L1 C/A code signal.

In this work, we extend this evaluation to the new Galileo signals and investigate their impact on a future multi frequency, multi constellation GBAS. Characterization of these new signals is based on ground subsystem measurements, since no flight data with GPS L5 or Galileo measurements are available at the moment. We assume that the improvements observed by ground receivers are also applicable to airborne measurements.
This assumption will be validated as soon as flight data are available.

The measurements used were collected from the DLR GBAS test bed over 10 days (note that Galileo satellite ground track repeatability is 10 sidereal days) between the $14^{\text {th }}$ and $23^{\text {rd }}$ of December 2013. In that period, four Galileo and four Block IIF GPS satellites were operational and broadcast signals on both aeronautical bands E1 / L1 and E5a / L5.

In Figure 3., the suppression of multipath and noise on the Galileo signals can be observed, where the code multipath and noise versus elevation for GPS L1 C/A, Galileo E1 (BOC) and Galileo E5a (BPSK(10)) signals are shown. The code multipath and noise was estimated using the linear dual frequency combination described in equation (10), where $M P_{i}$ represents the code multipath and noise on frequency $i, \rho_{i}$ the code measurement, and $\phi_{i}$, and $\phi_{j}$ represent the carrier-phase measurements on frequencies $i$ and $j$, respectively. Recall that carrier phase noises are small and can be neglected.

$M P_{i}=\rho_{i}-\left(1+\frac{2}{\alpha-1}\right) \phi_{i}+\left(\frac{2}{\alpha-1}\right) \phi_{j}$

The multipath on the Galileo E1 (BOC $(1,1))$ signal (the magenta curve) is lower than the GPS BPSK(1) on L1 (black curve), especially for low elevation, where the advantage of the E1 BOC $(1,1)$ is more pronounced. The lower values can be explained by the wider transmission bandwidth on E1 and the structure of the BOC signal [3]. Galileo E5a (green data in Figure 3) again shows a better performance than Galileo E1. This was expected due to the higher chip rate and higher signal power.

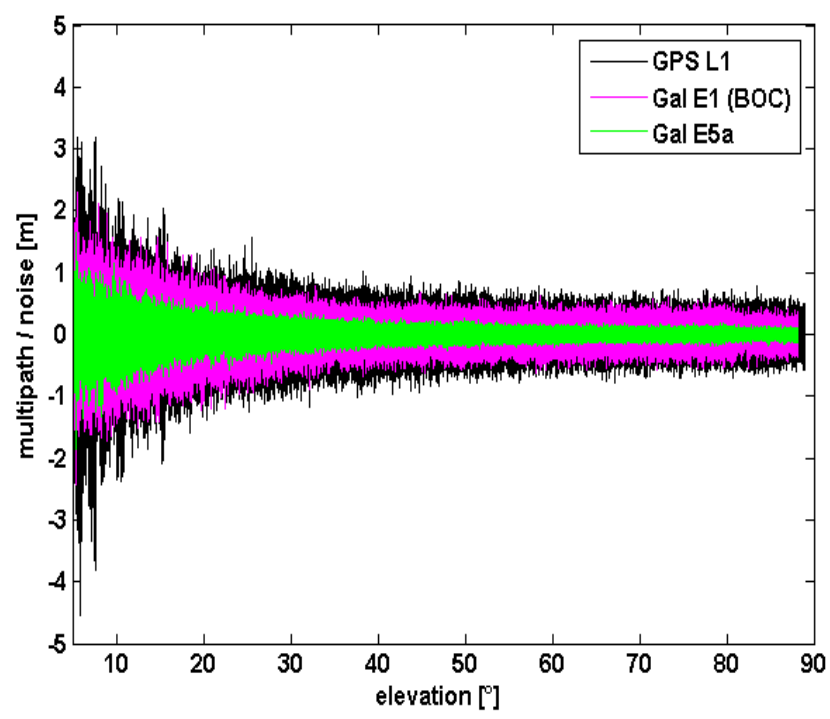

Figure 3. Raw multipath function of elevation for GPS L1, Galileo E1 (BOC $(1,1))$ and Galileo E5a (BPSK(10)) signals

A comparison of the multipath standard deviations for GPS L1, L5 and Galileo E1, E5a signals is presented in 
Figure 4. The standard deviations are computed by sorting the data into elevation bins with a spacing of $1^{\circ}$. The curves then show the ratio of the standard deviations for the elevation bins. The values for GPS L1 are almost 1.5 times larger than those for Galileo E1 BOC $(1,1)$ (green curve) for elevations below $20^{\circ}$. For high elevations, the ratio approaches 1.0. This corresponds to the observations in the raw multipath plot ( Figure 3). With the same signal modulation and the same chip rate, E5a and L5 have very similar results (red curve), and the ratio stays close to 1.0 for all elevations.

The blue and the purple curves in Figure 4 show the ratio of GPS L1 C/A (BPSK(1)) and GPS L5 (BPSK(10)), and Galileo E1 and Galileo E5a, respectively. The ratio of GPS L1 to GPS L5 (blue curve) increases with elevation from values around 2.5 for low elevations, reaching values above 3.5 for elevations higher than $60^{\circ}$. As Galileo E1 performs better, the ratio between Galileo E1 and Galileo E5a (purple curve) is smaller, from a value of 1.5 for elevations below 10 degrees to a value of 3.0 for high elevations.

Until now, we have presented the evaluation of raw code noise and multipath. However, in GBAS, carrier smoothing is performed to minimize the effect of code noise and multipath. The value which describes the noise introduced by the ground station is described by a standard deviation called $\sigma_{p r_{\text {_gnd }}}$ and is computed based on the smoothed pseudoranges from the reference receivers. In the following section, we focus on the evaluation of $\sigma_{p r_{-} g n d}$ using different signals and different smoothing time constants. Note that, in this study, $\sigma_{p r_{-} g n d}$ contains only smoothed multipath and noise; no other contributions (e.g., inflation due to signal deformation or geometry screening) are considered.

\section{$\underline{\text { B-values and } \sigma_{p r \text { gnd }}}$}

B-values represent estimates of the associated noise with the pseudorange corrections provided from each receiver for each satellite, as described in ED - 114A [2] and DO253C [8]. They are used to detect faulty measurements in the ground system. For each satellite-receiver pair $B(i, j)$, they are computed as:

$$
B(i, j)=P R C_{T X}(i)-\frac{1}{M(i)-1} \sum_{k \neq j} P R C_{S C A}(i, k)
$$

where $P R C_{T X}$ represents the candidate transmitted pseudorange correction for satellite $i$ (computed as an average over all $M(i)$ receivers), and $P R C_{S C A}$ represents the correction for satellite $i$ from receiver $k$ after smoothed clock adjustment, which is the process of removing the individual receiver clock bias from each reference receiver and all other common errors from the corrections. The summation computes the average correction over all $M(i)$ receivers except receiver $j$. This allows detection and exclusion of receiver $j$ if it is faulty. I If all B-values are below their thresholds, the candidate

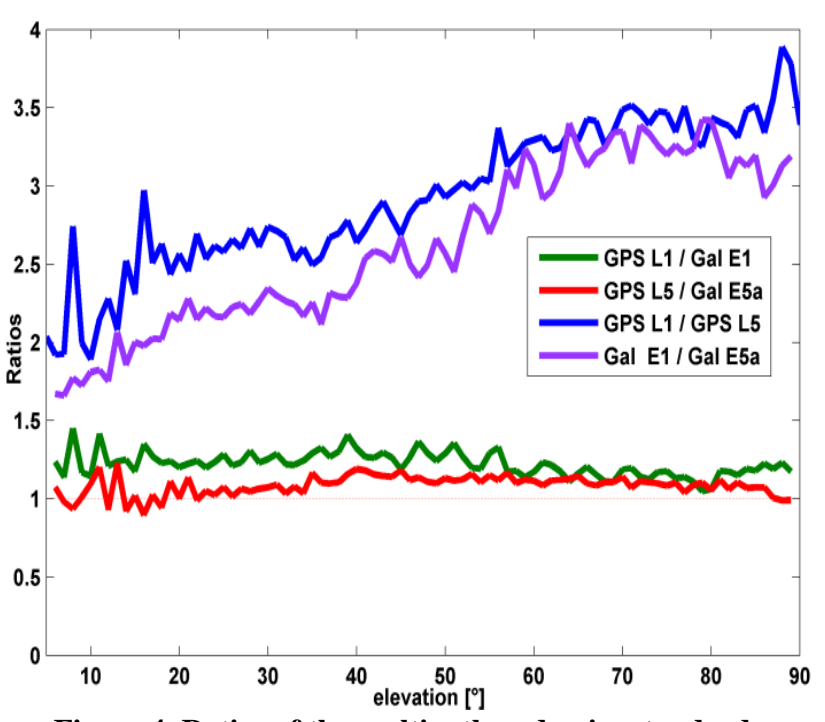

Figure 4. Ratios of the multipath and noise standard deviation function of elevation

pseudorange correction $\quad P R C_{T X}$ is approved and transmitted. If not, a series of measurement exclusions and $P R C$ and B-value recalculations takes place until all revised B-values are below threshold. Note that, under nominal conditions using only single frequency measurements, the B-values are mainly affected by code multipath and noise.

Under the assumption that multipath errors are uncorrelated across reference receivers, nominal B-values can be used to assess the accuracy of the ground system. The standard deviation of the uncertainty associated with the contribution of the corrections $\left(\sigma_{p r_{-} g n d}\right)$ for each receiver $m$ is related to the standard deviation of the B values by:

$$
\sigma_{p r_{\text {gnd }}, m}^{2}=\sigma_{B}^{2} \frac{(M-1)(N+1)}{N}
$$

where $M$ represents the number of the receivers and $N$ represents the number of satellites used. The final sigma takes into account the contribution from all receivers and is computed as the root mean square of the standard deviation of the uncertainties associated with each receiver (Eq. 12).

Figure 5 shows the evaluation of $\sigma_{p r_{-} g n d}$ for the Galileo E1, BOC $(1,1)$ signal and the GPS L1 C/A signal for increasing smoothing time constants $(10,30,60$, and 100 seconds). Starting with a 10 -second smoothing constant, Galileo E1 shows much better performance than GPS L1. The difference shrinks as the smoothing constant increases due to the effectiveness of smoothing in reducing noise and short-delay multipath. However, even with 100-second smoothing (the purple curves), Galileo E1 BOC $(1,1)$ shows lower values of $\sigma_{p r_{-} \text {gnd }}$.

A similar comparison is presented in Figure 6, where the performance of GPS L1 and Galileo E5a is compared. The Galileo E5a signal is significantly less affected by multipath, and the difference stays more pronounced than in the Galileo E1 - GPS L1, even with 100-second 
smoothing. It can be also observed that the Galileo signals have a lower sensitivity to the smoothing constant. The Galileo E1 signal shows an increase of sensitivity for low elevations (below $40^{\circ}$ ), while on E5a, a smoothing constant larger than 10 seconds has almost no impact on the residual error. Thus, a shorter smoothing constant on Galileo E5a generates approximately the same residual performance advantage of the new signals a decrease of the smoothing constant is one option for future applications. This would reduce the time required (for smoothing to converge) before including a new satellite or re-including a satellite after it was lost.

In the current GAST-D implementation, based on GPS L1 only, guidance is developed based on a 30-second

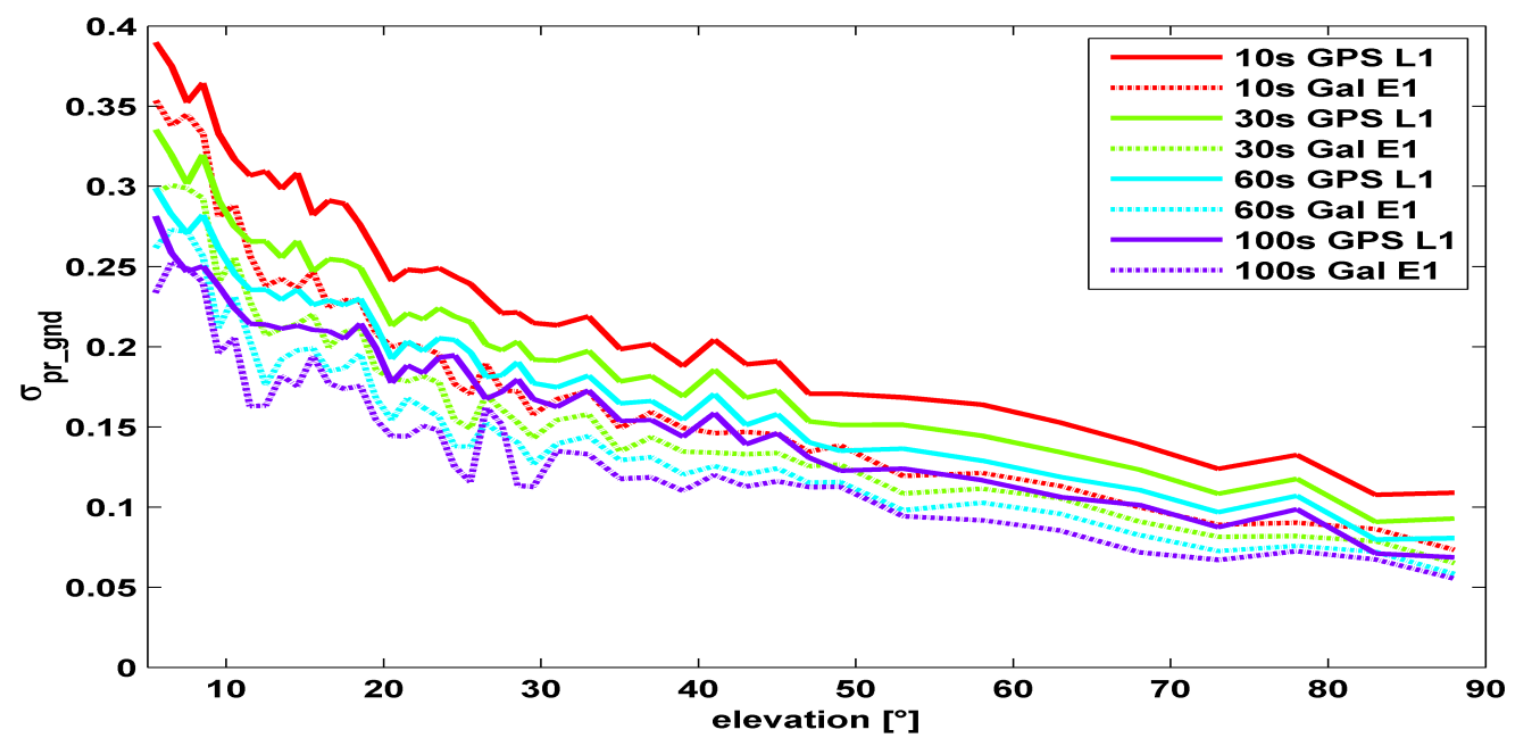

Figure 5. $\sigma_{p r_{-g} \text { gnd }}$ versus elevation for Galileo E1 (dashed lines) and GPS L1 (solid lines) for different smoothing constants: red (10s), green (30s), cyan (60s), purple (100s)

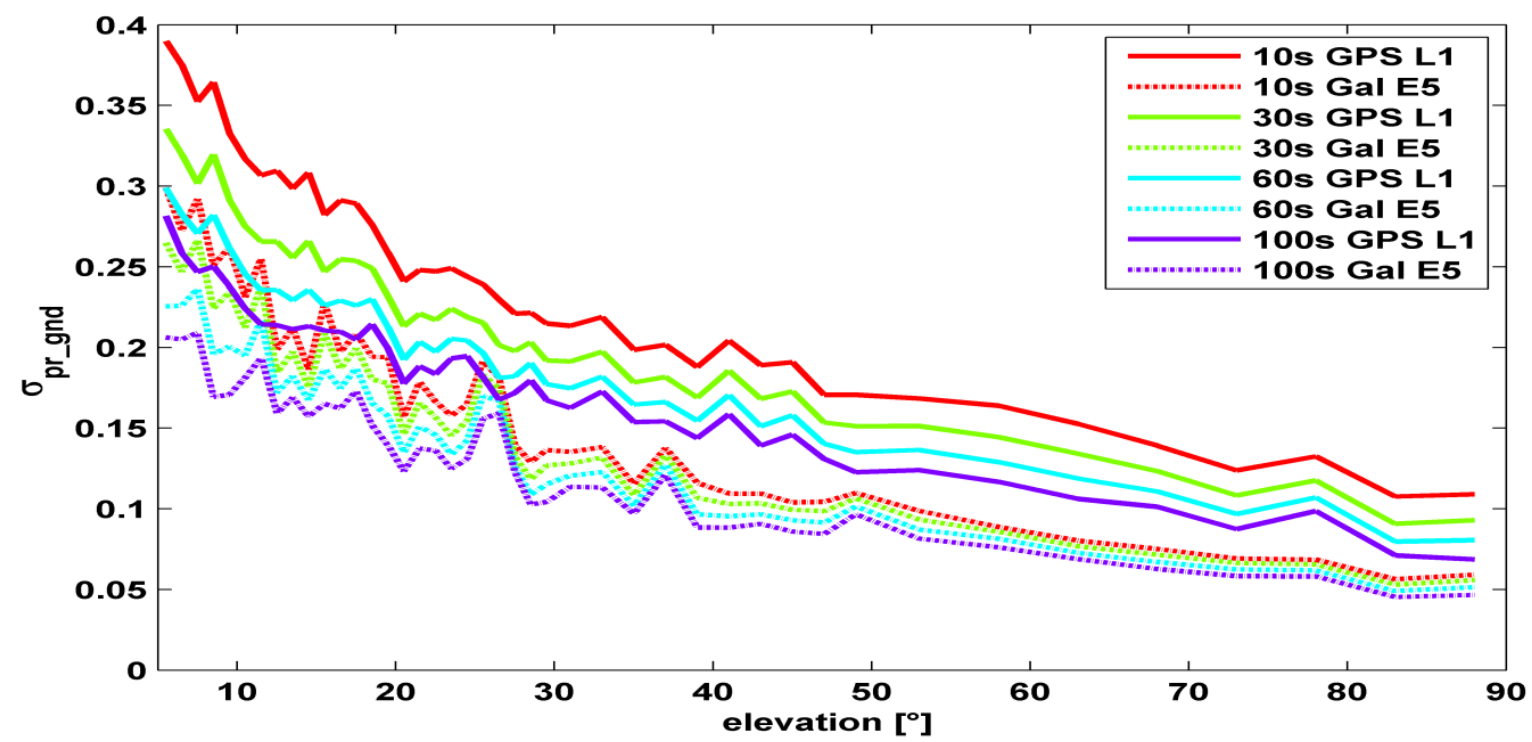

Figure 6. $\sigma_{p r_{-} g n d}$ versus elevation for Galileo E5a (dashed lines) and GPS L1 (solid lines) for different smoothing constants: red (10s), green (30s), cyan (60s), purple (100s)

noise and multipath as a 100 -second smoothing constant on GPS L1.

The values for $\sigma_{p r_{-} g n d}$ are, however, impacted by the number of satellites which are used to determine a correction. Since only a very limited number of satellites broadcasting L5 and Galileo signals are currently available, these results should be considered preliminary. However, they strongly indicate that with the new signals, we get better ranging performance. Based on the smoothing time constant. A second solution, one with 100 seconds of smoothing, is used for deriving the $D_{v}$ and $D_{1}$ parameters from the DSIGMA monitor [8] and thus for protection level bounding (it is also used for guidance in GAST-C). During the flight, different flight maneuvers or the blockage by the airframe can lead to the loss of satellite signals.

Figure 7 , shows the ground track of a recent flight trial conducted by DLR in November 2014. The colors represent the difference between the number of satellites 
used by the ground subsystem (with available corrections) and the number of satellites used by the airborne subsystem in the GAST-D position solution. One of the purposes of the flight was to characterize the loss of satellite signals in turns. In turns with a steeper bank angle, up to 3 satellites are lost (Turns 1, 3, and 4), while

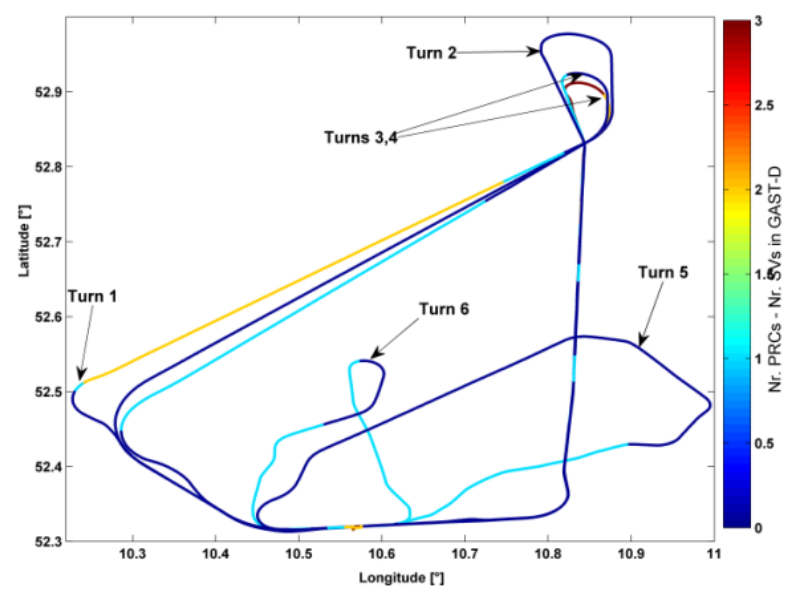

Figure 7. Ground track of a flight trial conducted by DLR. The colors represent difference between number of SVs used by the ground system and number of SVs used by the airborne

on a wide turn with a small bank angle (Turn 2), no loss of satellite lock occurred. It is also possible for airframe to block satellite signals, leading to a different number of satellites between ground and airborne even without turns. With this in mind, a shorter smoothing constant would allow the satellites lost to turns or to airframe blockage to be re-included more rapidly in the position solution. However, a new smoothing constant would have to be validated with a larger amount of data. Data from flights trials has to be evaluated as well to confirm that the same performances are valid also for the air multipath and noise.

In a future dual-frequency GBAS implementation, an important advantage of lower multipath and noise is to improve the Ifree position solution. In our previous paper [6], we demonstrated that the error level of the Dfree solution is almost the same as for single frequency, but an increase in error by a factor of 2.33 was computed for the Ifree standard deviation based on L1 C/A code and L2 semi-codeless measurements.

If the errors on L1 (E1) and L5 (E5a) code and carrier phase measurements are statistically independent the standard deviation of the $\sigma_{\text {Ifree }}$ can be written as

$$
\sigma_{\text {Ifree }}=\sqrt{\left(1-\frac{1}{\alpha}\right)^{2} \sigma_{L 1}^{2}+\frac{1}{\alpha^{2}} \sigma_{L 5}^{2}}
$$

where $\alpha=1-f_{1}^{2} / f_{5}^{2}$, and $\sigma_{L 1}, \sigma_{L 5}$ represent the standard deviations of the smoothed noise and multipath for L1 and L5, respectively. In a previous study [6], we observed that, despite having different raw multipath characteristics, the values of 100-second smoothed $\sigma_{p r_{\text {_gnd }}}$ on L1 and L5 are very similar. Considering $\sigma_{p r_{g n d}, L 1}=\sigma_{p r_{g n d}, L 5}$ in equation (13), the noise and multipath error on Ifree ( $\left.\sigma_{\text {Ifree }}\right)$ increases by a factor of 2.59 .

Figure 8 shows the ratio $\sigma_{\text {Ifree }} / \sigma_{L 1}$ using measured data. We observe that the measured ratio (the black curve) is below the theoretical ratio computed based on the assumption of statistically independent samples (the constant value of 2.59). This is explained by the fact that the multipath errors in the measurements are not independent but have some degree of statistical correlation. The standard deviations are computed based on the same data set used in the raw multipath and noise assessment using 100-second smoothed measurements sorted into elevation bins of $10^{\circ}$ spacing.

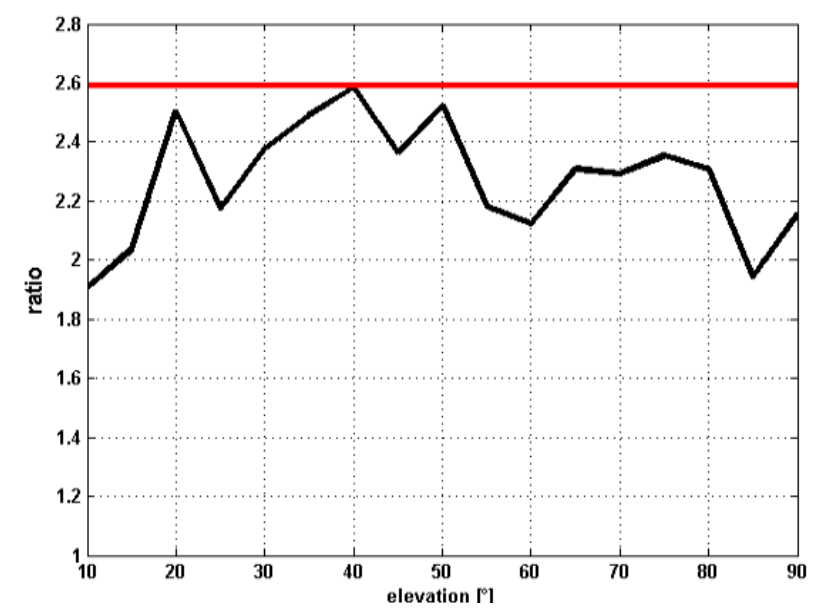

Figure 8. Measured ratio $\frac{\sigma_{I f r e e}}{\sigma_{L 1}}$ function of elevation

\section{INTER-FREQUENCY BIASES AND CORRECTIONS}

Inter-frequency bias can be defined as the difference of the mean of the code phase tracking error between any two signals received on any two frequencies. Note that, in this work, the carrier-phase inter-frequency bias is assumed to be negligible. This bias affects dual frequency signal processing and positioning in the GNSS receiver. When the inter-frequency bias is different with respect to different GNSS satellites, the resulting errors need to be carefully analyzed, as this residual bias cannot be absorbed into the clock offset estimate generated by positioning estimation. Satellite characteristics or distortions with respect to the signals as defined in the interface control documents (ICD) of the different GNSS systems introduce different tracking biases dependent on the antenna, front-end, and signal processing in the receiver. With respect to the antenna and front-end, amplitude response as well as group delay variations are to be considered. Concerning the signal processing parameters of a standard GNSS receiver, the one-sided receiver bandwidth $B$, the one-sided correlator spacing $\Delta$, and the discriminator or correlator type introduce different tracking biases and also inter-frequency biases. 
Signal distortions introduced by the satellite payload can be modeled as a combination of analog and digital distortions. This kind of classification can be considered for both nominal and non-nominal signal distortions. Digital distortion is a non-linear distortion that changes the actual chip duration of the transmitted signal. Analog distortion can be modeled as a linear distortion which accounts for all linear distortions introduced by the satellite payload (filtering effects). Such a classification is also considered by the International Civil Aviation Organization (ICAO), through its GNSS Working Group $\mathrm{B}$, which set forth classes of signal deformations or distortions for GPS satellites against which any candidate differential GNSS-based precision approach and landing system must be able to protect its users. These deformations/distortions are classified into 3 threat models [5]: threat model A, threat model B and threat model C. Threat model A includes only digital distortion, threat model B includes only analog distortion, and threat model $\mathrm{C}$ includes both digital and analog distortions.

These signal distortions can be determined from measurements using a high gain antenna as performed in [9], and the resulting tracking error bias and thus interfrequency bias can be derived following the methods outlined in [9]. In general, inter-frequency biases are different for different satellites and are different for different constellations (e.g., GPS and Galileo), as analog and digital distortions for different payloads and different frequencies are non-identical. However, using the measurements and methods described in [9], interfrequency biases for different satellites can be derived up to a residual uncertainty given by the accuracy of the measurements and of the models applied in the estimation process. This uncertainty needs to be assessed in detail starting from the derivation of measurement noise up to the estimation results. In [9], a maximum likelihood estimator, which can be considered asymptotically unbiased, is used for the joint estimation of digital and analog distortion. Moreover, assuming that the measurement errors are Gaussian (or overbounded by Gaussian), the estimation error also can be overbounded by a Gaussian distribution with a mean of the true parameter values and a variance of the inverse of the Fisher information with respect to the parameters to be estimated. Thus, also the uncertainty in the derived biases can be assumed to be Gaussian.

As an example, Table 2 shows the resulting biases induced by signal distortions for the L5 signals transmitted by the GPS satellites SVN 66 and SVN 68. According to the analysis in [9], the bias depends on the type of delay-locked loop (DLL) that is used by the receiver as well as on the receiver's front-end bandwidth $B$ and code correlator spacing $\Delta$. All values for bandwidth and correlator spacing are given as one-sided. For the non-coherent double delta DLL, $\Delta$ refers to the inner onesided correlator spacing. Note the different signs of the bias for different satellites. Since the biases are not equal, they cannot simply be attributed to receiver clock error and will degrade the position solution.

Table 2. Tracking bias [m] due to digital and analog distorsion

\begin{tabular}{|c|c|c|c|c|c|}
\hline \multirow{2}{*}{ Satellite } & \multirow{2}{*}{ PRN } & \multicolumn{2}{|c|}{$\begin{array}{c}\text { Coherent } \\
\text { DLL } \\
\text { B=12MHz, } \\
\Delta=\mathbf{0 . 5 c h i p s}\end{array}$} & \multicolumn{2}{c|}{$\begin{array}{c}\text { Non-coh. } \Delta \Delta \\
\text { DLL } \\
\text { B=12MHz, } \\
\Delta=\mathbf{0 . 0 5} \text { chips }\end{array}$} \\
\cline { 3 - 6 } & & Data & Pilot & Data & Pilot \\
\hline NAVSTAR 66 & 1 & +0.96 & +1.40 & +1.10 & +1.49 \\
\hline NAVSTAR 68 & 27 & -0.71 & -0.59 & -0.59 & -0.46 \\
\hline
\end{tabular}

\section{INCLUSION OF RESIDUAL ERROR IN GROUND SYSTEM CHARACTERIZATION}

As described in the previous section, inter-frequency bias depends on the specifics of each receiver and is a deterministic quantity which can be evaluated for a given hardware setup in GBAS reference receivers and airborne implementations. Since ground and airborne equipment will be different, the inter-frequency bias cannot be removed by differential corrections. Furthermore, since the biases differ between satellites, they will not be completely absorbed in the user clock. In Section 3, a detailed description of the dual-frequency smoothing techniques has been provided, and from equations 5 and 8 , we noted that only the Ifree solution is affected by the inter-frequency bias. For Ifree processing, we therefore propose the following:

- Removal of inter-frequency biases from the ground and airborne measurements independently

- Characterization of the residual uncertainty associated with each of the bias removals

- After bias removal, the residual uncertainties should be included in $\sigma_{p r_{-} g n d}$ (for the ground) and $\sigma_{p r_{-} a i r}$ (for the airborne).

As was described in the previous section, uncertainty in the bias removal process can be over-bounded by a Gaussian distribution which facilitates the inclusion of residual errors into $\sigma_{p r_{-} g n d}$ and $\sigma_{p r_{\_} a i r}$.

\section{CONCLUSION}

In this paper, we showed how GBAS can benefit from the new signals provided by the latest generation of GPS and Galileo satellites. We have demonstrated improved performance in terms of lower noise and multipath in data collected in our GBAS test bed. When GBAS is extended to a multi-frequency and multi-constellation system, these improvements can be leveraged for improved availability and better robustness of GBAS against ionospheric and other disturbances. 
The use of a second frequency, however, also brings new aspects to be considered. One is the introduction of interfrequency biases into the measurements. Depending on the hardware setup and receiver characteristics, these can be determined in advance and removed from the corrections generated by the ground system and from the measurements of the airborne receiver. A residual uncertainty associated with this process of bias removal should be included in the protection level for integrity assessments. We proposed to do this by incorporating these uncertainties into $\sigma_{p r_{-} g n d}$ and $\sigma_{p r_{-} a i r}$.

\section{ACKNOWLEDGMENTS}

Large portions of this work were conducted in the frame of SESAR under the associated partnership of AT-One.

\section{REFERENCES}

[1] A. Simsky, et al. "Experimental Results for the Multipath Performance if Galileo Signals Transmitted by GIOVE-A Satellite” International Journal of Navigation and Observation, Volume 2008, Article ID 416380

[2] Eurocae ED-114A, "Minimum operational performance specification for global navigation satellite ground based augmentation system ground equipment to support category I operations”, France, 2013

[3] Galileo OS SIS ICD Issue 1 Revision 1 September 2010e

[4] H. Konno, (2006) , “ Evaluation of Two Types of Dual-Frequency Differential GPS Techniques under Anomalous Ionosphere Conditions”

[5] International Civil Aviation Organization (ICAO), International standards and recommended practices, November 2001

[6] M.-S. Circiu, et al, "Evaluation of Dual Frequency GBAS Performance using Flight Data”, Proceedings of ION International Technical Meeting, San Diego, CA, January 2014

[7] P. Hwang, et al, "Enhanced Differential GPS Carrier-Smoothed Code Processing Using DualFrequency Measurements”, Navigation, J. of the Inst. of Navigation, Vol. 46, No. 2., 1999

[8] RTCA DO-253C, "Minimum operational performance standards for GPS local area augmentation system airborne equipment”, Tech. Rep. DO-253C, RTCA, Washington, 2008

[9] S. Thoelert et al., "Characterization of Nominal Signal Distortions and Impact on Receiver Performance for GPS (IIF) L5 and Galileo (IOV) E1 /E5a Signals”, ION GNSS 2014, Tampa, FL, U.S.A., September, 2014.
[10] T. Dautermann, M. Felux, A. Grosh , “ Approach service type $D$ evaluation of the DLR GBAS testbed”, GPS Solution, 2011, 2011, DOI 10.1007/s10291-011-0239-3 